\title{
The Application and Development of Statistical Technology in the New Times
}

\author{
Zhou Yan \\ Tianjin University of Finance and Economics, Changzhi, Shanxi Province, China \\ yanyanzhou489@163.com
}

Keywords: Economic statistics, New normal of statistics, Big data, Data mining

\begin{abstract}
With China's economic development stepping into the new normal, the statistics used to reflect the state of economic life also entered into the new normal. The domestic economic market has also been further improved and developed, which not only provides opportunities for China's statistical further development of the field, but also brings serious challenges. Data mining technology, a new statistical method, can be used to analyse the social and economic activities, and has been widely concerned by all circles of society. Using data mining technology to analyse data can meet the use of information needs of the users and promote sound and fast economic development in China.
\end{abstract}

\section{The Significance of Economic Statistics Application}

In the context of the gradual deepening of global economic integration, economic activities relates to more and more industry sectors, and involving the amount of information is also more and more huge. Based on such a scale, it is bound to bring a severe test to China's economic research activities. As we all know, data is the core form of information on the economic field. On the economic front, most of the funds are calculated and integrated, so its problem can also use the data to carry out analysis and processing. And this series of massive information flow, only the application of statistical methods to carry out systematic analysis, can only be converted into more accurate and comprehensive data vouchers, and provide good services for the follow-up economic construction.

Statistical methods can be applied to economic research activities, economic research activities in all links to get a lot of relevant data for basic security, means that economic construction under the application of economic statistics ${ }^{[1]}$ has a very important significance. If there is no statistical data support, economic research activities will be difficult to carry out smoothly. Economic statistics of a series of analysis and research are closely related to accurate data, so economic research should be carried out during the scientific and rational application of economic statistics. The effective application of economic statistics means that economic research activities, as well as the effective development of national economic activities.

\section{The application of economic statistics}

In the current context of national economic construction and economic statistics in the construction process of the actual application of the situation, we can say that in China's economic construction and research areas, the application of economic statistics ${ }^{[2]}$ is still not deep enough, and 
has been stranded in the preliminary understanding and the initial stage of application. If we want to better serve economic statistics in the country's economic construction and research work, the state needs to first collection of outstanding education and research personnel, composed of research and investigation team, to work together to find economic statistics to enhance the effectiveness and effectiveness of measures. In addition, if we want to make economic statistics play a more significant supporting role in economic research and construction activities, we must first find out the key and difficult problems in China's current economic construction links, and with the help of further research and analysis, we can achieve the use of economic statistics to improve the economic situation and solve economic problems. Only in this way can the economic construction work better adapt to the development trend of the times and development needs, and ensure that economic statistics can make a more outstanding contribution to the country's economic construction.

In the context of China's current economic development, the community for economic statistics in the economic construction and other aspects of the application requirements have always been improved. However, the majority of enterprises for the application of economic statistics and research is still stuck in the initial stage and has not been in-depth. On the application of economic statistics, we need the relevant experts and scholars to conduct more in-depth research and thinking, and then to make economic statistics in China's economic construction links, so that their support and support role to play better, which is also one of the most important issues in the field of economic research and construction. Since China entered a new stage of development, the majority of domestic economic statistical management system has been completely unable to adapt to the current domestic economic market sustainable development requirements. This can be seen that the development of domestic economic statistics foundation is not strong. But in the process of sustainable development, through the management and research links to further strengthen this problem has been a more obvious improvement in recent years.

In recent years, many well-known experts and scholars in the field of economics ${ }^{[3]}$, as well as managers and professional staff of the financial departments of many large domestic enterprises, have begun to realize the important role of statistical methods in solving internal financial problems. They began to explore the financial issues among the relevant statistical content. The results of the research fully prove that the application of economic statistics in the internal economic management activities is to enable enterprises to find financial risks as soon as possible and to find an effective way to enable enterprises to solve these hidden dangers as soon as possible, which can also provide a more efficient means for the forward-looking analysis of financial related issues and decisionmaking and has very obvious promoting effect for enterprise management level and financial income of upgrade.

\section{New Normal}

With the gradual deepening of reform and opening and the rapid development of market economy, China's economy has gradually entered the new normal. In this new era of economic transformation and development, the statistical work for the economy can provide a more comprehensive and accurate data support for economic development on the new situation of its own development. But whether it can play its own unique advantages in the new normal, it has been become a new problem.

\subsection{The meaning of New Normal of Statistics}

Central China's current economic development law is defined as the new normal, and clearly pointed out that all departments should actively take the initiative to adapt to the new normal. The statistics as a service for the economic life of the work and statistical data as an important indicator 
of economic life, can adapt to the new normal changes with the economic development. New normal of statistics is the new statistics that can correctly reflect the new normal of economy.

\subsection{The necessity of New Normal of Statistics}

The new normal of statistics ${ }^{[4]}$ is inevitable when the economy enters the new normal state. When facing such a rapidly changing economic environment, the original statistical work attitude has been unable to adapt to the new situation of economic life. Therefore, the statistical work of the new normal change is the inevitable development of the times demands and choices. At the same time, with the change of social environment and the coming of the information age, statistics is no longer just the unique needs of government departments, all sectors of society and enterprises have put forward the demand for statistics, so the community agencies to supplement government statistics has been become a new situation. In addition, emerging technologies also provide new technical support for statistical work and the possibility of new normal development. The relevant policies issued by the state and the cooperation of various social institutions set up a platform for the development of new normal statistics.

\subsection{The main performance of New Normal of Statistics}

The statistical work reflects the changes in economic and social life development, which also can provide support for the implementation of government management and policy support. Starting from the 18th National Congress, the state has attached more importance to the work of statistics. Many leaders have elaborated on the importance of statistical indicators at the meeting and clearly pointed out that they should give full play to the role and contribution of statistical work. In such a high concern, the statistical data is also concerned about the correctness. Often some of the data published with the social questioning voice, in the new normal economy, the statistics are often subject to the parties questioned. Faced with so many questions, the relevant statistical standards introduced by the state will be further transparent and statistical data will accept the supervision of all parties. In the new normalization of the economy, China's economy is facing new problems such as under-consumption and irrational industrial structure. At the same time, it faces new unforeseen problems in the new economy. To solve these problems, it is necessary to support the statistical data. Statistical work has brought enormous tasks and challenges, and the work of statistics has become increasingly arduous. With the continuous innovation of information technology and the arrival of large data era, the combination of statistical work and large data is the only way. Big data for the statistical work brings a large number of accurate and scientific data sources, and improves statistical forecasting ability. And large data application of statistical change is the most important.

\section{The normalization of big data statistics}

\subsection{Establishing an awareness of big data}

The arrival of the big data era is the inevitable social development. In the era of big data, information sharing and circulation is larger, and the volume of information is larger, and the statistical work is more complex, the statistical staff of professional and professional requirements are also higher. Therefore, the statisticians must fully understand the importance of statistical system and method reform in large data age, establish the consciousness of large data, profoundly understand the connotation and significance of information statistics in the era of big data, grasp the overall characteristics of large data. So as to better do a good job in statistical work in the big data age. 


\subsection{Improving data sharing}

Big data age is a significant feature of sharing information ${ }^{[5]}$. In the era of large data, sharing reflects the maximum value of the data. The current statistical system is mainly in accordance with regional, professional and industry, the effective utilization of information is not high. However, in the era of large data, information utilization greatly improved, while data integration is also strong. Therefore, for the statistical data, it should be an effective exchange and sharing, so that data information can become a common social resource. We also can enhance the sharing of data and effective utilization so that to provide more decision-making value for China's economic development.

\subsection{Training big data talents}

The arrival of the big data era makes the statistical system and statistical methods have changed. The statistical talent must be put forward higher requirements, and the statistical personnel should also meet the needs of large data age. Therefore, we should strengthen the training of statistical personnel, specifically from the operational capacity, professional quality, professional knowledge and other aspects of training, so that statisticians not only have a profound statistical theory, but also have strong data collection, processing, and analysis skills. We can also make large data better applied to the statistical work, so that more talents can translate from statistical experts to statistical data experts and data analysts. These experts can make a clear analysis and grasp to the large data, so as to improve the scientific and scientific rationality.

\section{The Application of Data Mining in Economic Statistics}

\subsection{Data preprocessing}

In the process of economic statistics, preprocessing is one of the most basic way. Mining technology ${ }^{[6]}$ itself is a technology which provides basic information as a basis and make intelligent analysis of information. In the use of the process, because it will subject to economic information constraints, it can not occur out of nothing. Therefore, all the economic statistics data need to be pretreated. The main contents of preprocessing are: the data is uncertain, inaccurate and not true, but also need to calculate the difference between economic statistics.

On the basis of these basic data to deal with the problem, this process is also known as data cleansing. At present, the current use of data cleaning methods are the mean method, smoothing method and forecasting method. In these methods, the mean method is an application form of the fuzzy concept in the current analysis technology. If there is a data point in the basic data is null or the data point is noise data, the mean method can be used to deal with it, which is to use all of this attribute mean to fill the vacancy. Only in this way can provide a guarantee for the smooth making of the basic data analysis and collation, and ultimately to obtain more accurate analysis of data. The smoothing method is mainly based on the existence of the null data and noise data, and the mean difference between the method is that the use of weighted average smoothing method instead of the average. This takes full account of the calculation of each extraction process. And the data on the results of the impact of the final calculated results are often closer to the real value.

\subsection{Neural Networks}

Neural network is a kind of intelligent information technology to simulate human brain information processing. And it is the same as human neural network process, which are both the process of information input, information analysis and information output, but neural networks are 
applied in the process of economic statistics. This method provides people with a relatively accurate and complete process, and then let the economic mode and people in the information to accept and process the same process, so that the process becomes more visual, concrete and practical. It can make a better contact in the economic process, and then make a reasonable analysis of economic issues, from which to get a better approach.

\subsection{Decision tree}

Decision tree is a kind of algorithm used in prediction model and a nonparametric identification technique based on statistical theory. It has the purpose of classifying large amounts of data and finding valuable potential information. It is precisely because its classification is fast and the description of the information is also very simple, decision tree can handle large-scale data of the economic operation.

\subsection{Genetic algorithm}

Genetic algorithm ${ }^{[7]}$ is mainly based on the natural and genetic mechanism of biological, and the algorithm is randomly selected. Its main idea is to combine with the social problems, and to collect information in the designated population, and to integrate and analyse with hidden information, and ultimately to get the results. Genetic algorithm is implicit, it is easy to combine with other models, and can collect hidden data, and then excavated data analysis, and ultimately be applied. And the economic problem belongs to a kind of development and change problem, its interior has the relatively complex connection. Genetic algorithm can be referenced from the source, and gradually extended down, and then extract the data in them, and the overall analysis of these data, which is to promote economic issues more specific, targeted and direct to a certain extent. This allows the relevant personnel to deal with the problem becoming more intuitive, and some of the hidden problems become more expression. In this way, to make statistical work become more simple and direct.

\section{Conclusion}

With the deepening of reform and opening, the market economy continues to deepen and perfect. In order to carry out in-depth exploration of economic construction, it is essential to rely on data support. Economic statistics provide data support for decision making and implementation, ensuring that economic activities are justified. The application of data mining technology in economic statistics can ensure the economic activities more comprehensive and concrete, and can guarantee the quality of economic statistics. Data mining technology has broad prospects for the development of society, which can create enormous economic benefits and enhance social value. In terms of the data mining's function, under the condition of economic construction, for the application of economic statistics, it must acquaint with its significance and impact, analyse detailed the problem that it will be confronted with, learn the advanced knowledge applied to practise constantly, keep pace with the time, make it spilt and present radiance.

\section{References}

[1] Ruini Liu. (2014) On the Application of Economic Statistics in the Background of Economic Construction. Enterprise Reform and Management, 18, 216-216.

[2] Weihong Xu. (2015) Application of Economic Statistics in Economic Construction. Financial Economics (Theory Edition), 129-131.

[3] Qinpei Hong. (2014) An Analysis of the Application of Economic Statistics in Economic Construction. Science and technology to get rich guide, 6, 242-242. 
[4] Xiufang Chen. (2015) Reflections on Statistical Work under New Norms. Inner Mongolia Statistics, 2, 61-62. [5] Qiang Shen. (2014) Reflections on the New Normal. Statistical Science and Practice, 12.

[6] Yue Yue. (2015) Discussion on the Application of Data Mining Technology in Economic Statistics. Operation and management, 30-198.

[7] Jing Dong. (2015) Analysis on the Application of Data Mining Technology in Economic Statistics. Business, $31,227-228$. 